\title{
PROPAGATION OF SINGULARITIES OF SOLUTIONS TO SEMILINEAR BOUNDARY VALUE PROBLEMS
}

\author{
FLORIN DAVID AND MARK WILLIAMS
}

\begin{abstract}
Let $P$ be a second-order, strictly hyperbolic differential operator on an open region $\Omega \subset \mathbf{R}^{n}(n \geq 3)$ with smooth noncharacteristic boundary. Given a solution $u \in H_{\mathrm{loc}}^{s}(\bar{\Omega}), s>(n+1) / 2$, to $P u=f(x, u)$, we discuss the propagation of microlocal $H^{r}$ singularities in the range $s \leq r<2 s-n / 2$ in the general case where the Hamilton field of $p$ may be tangent to $\partial T^{*} \Omega \backslash 0$ to arbitrarily high finite or infinite order.
\end{abstract}

Introduction. Recently, M. Sablé-Tougeron $[\mathbf{1 0}, \mathbf{1 1}]$ and A. Alabidi $[\mathbf{1}]$ used the calculus of paradifferential operators to obtain, for a quite general class of nonlinear boundary value problems, results describing the reflection of singularities that travel on bicharacteristics transversal to the boundary of a region $\Omega \subset \mathbf{R}^{n}$. Using a different approach, similar to the one initiated by Rauch [9] and further developed by Beals and Reed [3] in studying interior propagation, we have obtained results for second-order semilinear problems with Dirichlet conditions in the general case where tangential bicharacteristics as well as gliding rays may carry singularities. Our argument has two main steps. We first prove an analogue of Rauch's Lemma for a class of spaces measuring microlocal $H^{s}$ regularity up to the boundary. We also require a precise linear theorem (Theorem 2), a refinement of the results of Melrose and Sjöstrand ([8], see also [6]), which describes the propagation of $H^{s}$ regularity along generalized bicharacteristics. This theorem must apply to equations $P u=v$ for distributions $v$ which lie in our microlocal algebras, but which cannot be assumed to be normally regular. A simple inductive argument combining the above then yields the desired semilinear theorem (Theorem 1). Complete proofs will appear in [4].

Spaces of distributions near $\partial \Omega$. Let $\tilde{U}$ be a coordinate chart centered at $x_{0} \in \partial \Omega$, and let $\left(x_{1}, x^{\prime}\right)$ be coordinates in which $\bar{\Omega} \cap \tilde{U}(\equiv U)=\left\{x_{1} \geq 0\right\}$. We will use the spaces $H_{t, t^{\prime}}^{\text {loc }}(U)$ defined in Hörmander [5]. For $u \in H_{r,-\infty}^{\text {loc }}(U)$ we define $\widetilde{W F}_{r}(u) \subset\left(T^{*} \partial U \backslash 0\right) \cup\left(T^{*} \stackrel{\circ}{U} \backslash 0\right)$ as follows. If $\sigma \in T^{*} \partial U \backslash 0, \sigma \notin$ $\widetilde{W F}_{r} u$ if and only if for some tangential pseudodifferential operator $\phi\left(x, D^{\prime}\right)$ of order zero, elliptic at $(0, \sigma), \phi u \in H_{\text {loc }}^{r}(U)$. In this case we write $u \in \tilde{H}^{r}(\sigma)$. In $T^{*} \stackrel{\circ}{U}, \widetilde{W F}_{r}$ coincides with the usual notion of $W F_{r}$. Note that the definition of $\widetilde{W F}_{r} u$ is coordinate dependent. However, it is a consequence (see [4]) of the Lemma stated below and the fact that $\partial \Omega$ is noncharacteristic, that for

Received by the editors December 10, 1985.

1980 Mathematics Subject Classification. Primary 58G17, 35L70.

(C) 1986 American Mathematical Society $0273-0979 / 86 \$ 1.00+\$ .25$ per page 
solutions $u \in H_{\text {loc }}^{s}(\bar{\Omega}), s>(n+1) / 2$, to $P u=f(x, u), \widetilde{W F_{r}} u$ is coordinate independent for $s \leq r<2 s-n / 2+1 / 2$.

It was observed in $[\mathbf{1 0}]$ that the spaces $H_{t, t^{\prime}}^{\text {loc }}(U)$ are algebras stable under $f(x, u)$ if $t>\frac{1}{2}, t+t^{\prime}>n / 2$, and $t+2 t^{\prime}>\frac{1}{2}$. Thus, given $u \in H_{\text {loc }}^{s}$ such that $P u=f(x, u)$, Peetre's Theorem [5, 4.3.1] yields by induction that $u \in$ $H_{2 s-\rho+2,-s+\rho-2}^{\text {loc }}(U)$ for all $\rho>\frac{1}{2}$. This suggests considering, for $\sigma \in T^{*} \partial U \backslash 0$, the following spaces as candidates for microlocal algebras at the boundary:

$$
\mathcal{A}_{s, s^{\prime}, \rho}(\sigma)=H_{2 s-\rho,-s+\rho}^{\text {loc }}(U) \cap \tilde{H}^{s^{\prime}}(\sigma) .
$$

Main results. The following lemma is, we think, the natural analogue of Rauch's Lemma at the boundary.

LEMMA. The spaces $A_{s, s^{\prime}, \rho}(\sigma)$ defined above are algebras stable under the action of $C^{\infty}$ functions $f(x, u)$ provided $(n+1) / 2<s \leq s^{\prime}<2 s-n / 2-\rho$ and $\rho \in(1 / 2, s-n / 2)$.

In the statement of the following theorem we take $\Omega=\omega \times \mathbf{R}_{x_{n}}$, where $\omega \subset \mathbf{R}^{n-1}$ is an open region with smooth boundary. $\forall$ and $\mathcal{G}$ denote the hyperbolic and glancing regions of $T^{*} \partial \Omega \backslash 0$ (see $[\mathbf{8}$ or 6$]$ ).

THEOREM 1. Let $P$ be a second-order differential operator, noncharacteristic with respect to $\partial \Omega$, and strictly hyperbolic with respect to the surfaces $x_{n}=c$. Suppose that $u \in H_{\text {loc }}^{s}(\bar{\Omega}), s>(n+1) / 2$, satisfies $P u=f(x, u)$ in $\Omega$. Then for $s \leq r<2 s-n / 2$, every point $\gamma_{0} \in \widetilde{W F}_{r} u \backslash W F_{r}\left(\left.u\right|_{\partial \Omega}\right)$ is either a characteristic point of $P$ in $T^{*} \Omega$, or else contained in $\forall \cup \mathcal{G}$. An open interval $(-T, T) \ni t \rightarrow \gamma(t)$ with $\gamma(0)=\gamma_{0}$ on a generalized bicharacteristic $[\mathbf{8}, \mathbf{6}]$ is contained in $\widetilde{W F} r$.

Theorem 1 is proved by an inductive argument using the lemma and the following linear theorem. Note that the assumptions on $P$ in Theorem 1 and the coordinate invariance of $\widetilde{W F}_{r} u$ for $u \in H_{\text {loc }}^{s}$ satisfying $P u=f(x, u)(r \in$ $\left.\left[s, 2 s-n / 2+\frac{1}{2}\right)\right)$ allow us to reduce to the case where $P$ and $\Omega$ are as described in Theorem 2. We denote points in $T^{*} \mathbf{R}^{n}$ by $\left(x_{1}, x^{\prime}, \xi_{1}, \xi^{\prime}\right)$.

THEOREM 2. Suppose $\Omega \subset \mathbf{R}_{+}^{n}=\left\{x_{1}>0\right\}$ and write $\partial \Omega=\bar{\Omega} \cap \partial \mathbf{R}_{+}^{n}$. Let $P=D_{x_{1}}^{2}-R\left(x, D^{\prime}\right)$ be a second-order differential operator on $\Omega$ strictly hyperbolic with respect to the planes $x_{n}=c$. Suppose $P u=f$ in $\Omega$, where for some $s^{\prime} \geq 1, u \in H_{s^{\prime}+2,-\infty}^{\text {loc }}(\bar{\Omega}), f \in H_{s^{\prime},-\infty}^{\text {loc }}(\bar{\Omega})$, and $u \in H^{s^{\prime}+1}\left\{\left(x, \xi_{1}, 0\right): x_{1}>\right.$ $0, x$ near $\partial \Omega\}$. Then every point $\gamma_{0} \in \widetilde{W F}_{s^{\prime}+1 / 2} u \backslash\left(\widetilde{W F}_{s^{\prime}} f \cup W F_{s^{\prime}+1 / 2}\left(\left.u\right|_{\partial \Omega}\right)\right)$ is either a characteristic point of $P$ in $T^{*} \Omega$, or else contained in $\forall \cup \mathcal{G}$. An interval $(-T, T) \ni t \rightarrow \gamma(t)$ with $\gamma(0)=\gamma_{0}$ on a generalized bicharacteristic is contained in $\widetilde{W F}_{s^{\prime}+1 / 2} u$.

Sketch of the proofs. We begin with the lemma. Any $u \in A_{s, s^{\prime}, \rho}(\sigma)$ can be extended to an element $\tilde{u} \in H_{\text {loc }}^{s}(\tilde{U})$ such that $\tilde{u}=u_{1}+u_{2}$, where $u_{1} \in$ $H_{\text {loc }}^{s^{\prime}}(\tilde{U})$ and where $u_{2} \in H_{2 s-\rho,-s+\rho}^{\text {loc }}(\tilde{U})$ has the property that $\phi\left(x, D^{\prime}\right) u_{2} \in$ $H_{2 s-\rho, \infty}\left(\mathbf{R}^{n}\right)$ for $\phi\left(x, D^{\prime}\right)$ supported sufficiently near $(0, \sigma)$. Now given $u$ and $v$ in $A_{s, s^{\prime}, \rho}(\sigma)$, we first choose such extensions $\tilde{u}=u_{1}+u_{2}, \tilde{v}=v_{1}+v_{2}$. We then show that tangential operators of order zero of the form $\chi\left(D^{\prime}\right) \circ \psi^{2}(x)$, 
where $\psi \in C_{0}^{\infty}\left(\mathbf{R}^{n}\right)$, map $\tilde{u} \tilde{v}$ into $H^{s^{\prime}}\left(\mathbf{R}^{n}\right)$ provided they are supported sufficiently near $(0, \sigma)$. For $u_{1} v_{1}$ this is immediate, and the convolutions corresponding to the products $u_{1} v_{2}$ (or $u_{2} v_{1}$ ) and $u_{2} v_{2}$ are examined separately. The stability of the algebras under smooth $f(x, u)$ then follows by considering $D f(x, u)$, using the chain rule and an inductive argument like the one given in Beals [2, Corollary 1.8].

Next we will sketch an argument that can be used to prove Theorem 2 when, for example, $\gamma_{0} \in \mathcal{H}$ or $\gamma_{0} \in \mathcal{G}_{d}$ (the diffractive region). Say $\gamma_{0}=\sigma \in \mathcal{G}_{d}$, where the $x_{n}$-coordinate of $\sigma$ is 0 . Given $P u=f(x, u) \in \tilde{H}^{s^{\prime}}(\sigma),\left.u\right|_{\partial U} \in$ $H^{s^{\prime}+1 / 2}(\sigma), u \in H^{s^{\prime}+1 / 2}\left(\Gamma_{-}\right)$, where $\Gamma_{-} \subset T^{*} \stackrel{\circ}{U}$ is the portion in $x_{n}>-\varepsilon$ of the incoming tangential ray passing over $\sigma$, we must show $u \in \tilde{H}^{s^{\prime}+1 / 2}(\sigma)$. We can suppose $f \in H^{s^{\prime}}\left(\Gamma_{-}\right)$. By solving a mixed problem in $U$ we first find a distribution $w \in H_{\text {loc }}^{s^{\prime}+1 / 2}(U)$ such that $u^{\prime}=u-w$ satisfies $P u^{\prime}=f, \sigma \notin W F$ $\left.u^{\prime}\right|_{\partial U}, u^{\prime} \in H^{s^{\prime}+1}\left(\Gamma_{-}\right)$. Extend $f$ to an element $\tilde{f} \in H_{\boldsymbol{s}^{\prime},-\infty}^{\text {loc }}(\tilde{U})$ such that $\chi\left(x, D^{\prime}\right) \tilde{f} \in H^{s^{\prime}}\left(\mathbf{R}^{n}\right)$ for all $\chi \in L^{0}$ supported sufficiently near $(0, \sigma)$. Now solve, in $\tilde{U}$, the extension problem:

$$
P v=\tilde{f}, \quad v=u^{\prime} \quad \text { near } \Gamma_{-} \text {in } x_{n}<-\varepsilon / 2 .
$$

One then shows that $A\left(x, D^{\prime}\right) v \in H^{s^{\prime}+1}\left(\mathbf{R}^{n}\right)$ for all $A \in L^{0}$ supported sufficiently near $(0, \sigma)$. (It is obvious that $A v \in H^{s^{\prime}+1}\left(p^{-1}(0)\right)$ for such $A$.) Next choose such an $A \equiv 1$ near $(0, \sigma)$ and solve the mixed problem in $U$ :

$$
P w^{\prime}=0,\left.\quad w^{\prime}\right|_{\partial U}=\left.A\left(u^{\prime}-v\right)\right|_{\partial U}, \quad w^{\prime}=0 \text { in } x_{n}<-\varepsilon / 2 .
$$

Since $\left.A v\right|_{\partial U} \in H^{s^{\prime}+1 / 2}, w^{\prime} \in H_{\mathrm{loc}}^{s^{\prime}+1 / 2}(U)$. The results of Melrose [7] and Taylor [12] imply $\sigma \notin W F_{b}\left(u^{\prime}-\left(v+w^{\prime}\right)\right)$. Hence $u^{\prime} \in \tilde{H}^{s^{\prime}+1 / 2}(\sigma)$, which implies $u \in \tilde{H}^{s^{\prime}+1 / 2}(\sigma)$ as desired.

In cases of finite-order tangency where the incoming or outgoing generalized bicharacteristic is a gliding ray, and in the case of infinite-order tangency, our proof of Theorem 2 follows the arguments in Hörmander [6, §24.5] closely. Again, an appropriate mixed problem is solved first. The remainder of the proof involves checking that the $L^{2}$ pairings in $[6,24.5]$ remain bounded under the hypotheses of Theorem 2.

It is a pleasure to thank Richard Melrose for helpful conversations concerning the linear theorem, and for encouraging us to pursue the case of higher-order tangency.

NOTE ADDED IN PROOF. The proof of Theorem 2 has been simplified in [4]. A method similar to that sketched above for $\sigma \in \mathcal{G}_{d}$ can be used to handle all points $\sigma \in \forall \cup \mathcal{G}$. The revised argument allows $H^{r}$ regularity for $r<2 s-n / 2+\frac{1}{2}$ to be propagated in Theorem 1 .

\section{REFERENCES}

1. A. Alabidi, Réflexion transverse des singularités pour un problème aux limites non linéaire d'ordre 2, C.R. Acad. Sci. Paris 300 (1985), 287-290.

2. M. Beals, Propagation of smoothness for nonlinear second order strictly hyperbolic differential equations, preprint, 1985. 
3. M. Beals and M. Reed, Propagation of singularities for hyperbolic pseudodifferential operators with nonsmooth coefficients, Comm. Pure Appl. Math. 35 (1982), 169-184.

4. F. David and M. Williams, Singularities of solutions to semilinear boundary value problems (in preparation).

5. L. Hörmander, Linear partial differential operators, Springer-Verlag, Berlin, 1969.

6. __ The analysis of linear partial differential operators. III, Springer-Verlag, Berlin, 1985.

7. R. B. Melrose, Microlocal parametrices for diffractive boundary value problems, Duke Math. J. 42 (1975), 605-635.

8. R. B. Melrose and J. Sjöstrand, Singularities of boundary value problems. I, II, Comm. Pure Appl. Math. 31 (1978), 593-617; 35 (1982), 129-168.

9. J. Rauch, Singularities of solutions to semilinear wave equations, J. Math. Pures Appl. 58 (1979), 299-308.

10. M. Sablé-Tougeron, Paralinearisation de problèmes aux limites non linéaires, C. R. Acad. Sci. Paris 299 (1984), 169-171.

11. __ Regularité microlocale pour des problèmes aux limites non linéaires, C.R. Acad. Sci. Paris 300 (1985), 283-286.

12. M. E. Taylor, Grazing rays and reflection of singularities of solutions to wave equations, Comm. Pure Appl. Math. 29 (1976), 1-38.

Department of Mathematics, University of North Carolina, Chapel HiLl, NORTH CAROLINA 27514 